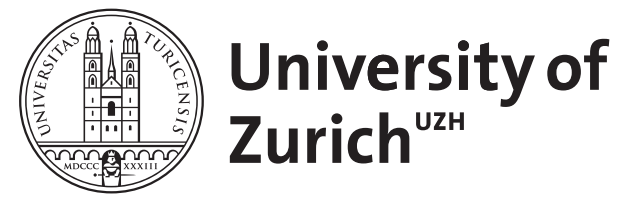

\title{
Sport helps to prevent strokes
}

Luft, A R

DOI: https://doi.org/10.2217/ahe.11.71

Posted at the Zurich Open Repository and Archive, University of Zurich ZORA URL: https://doi.org/10.5167/uzh-87447

Journal Article

Accepted Version

Originally published at:

Luft, A R (2011). Sport helps to prevent strokes. Aging Health, 7(6):801-802.

DOI: https://doi.org/10.2217/ahe.11.71 


\section{Sport helps to prevent strokes}

Lower prevalence of silent brain infarcts in the physically active: The Northern Manhattan Study by Willey, Moon, Paik, Yoshita, DeCarli, Sacco, Elkind and Wright

Neurology 2011, 76:2112-8

\section{Summary}

Physical inactivity is a risk factor for stroke. This population-based cohort study investigates the risk for subclinical brain infarction and chronic white matter changes measured by magnetic resonance imaging (MRI) in individuals that perform different levels of activity on a regular basis. The study finds the risk for infarction, but not for white matter changes to be significantly reduced in active subjects independent of classical risk factors. Activity, however, needs to be vigorous to be effective. The study supports to role of inactivity as an independent cerebrovascular risk factor. Physicians and health care providers should encourage their elderly patients to remain physically active.

\section{Keywords}

stroke, physical activity, MRI

\section{Summary of methods and results}

The study is based on the Northern Manhattan Study (NOMAS), a population-based prospective cohort study to investigate risk factors for cerebrovascular diseases. A subsample of the NOMAS cohort underwent MRI of the brain to enable the detection and quantification of subclinical brain infarcts (SBI, typically small infarctions of the lacunar type) and chronic white matter changes visible as white matter hyperintensities (WMH) on MRI. SBI were defined as lesions $\geq 3 \mathrm{~mm}$ in diameter with a CSF-filled cavitation that could not be attributed to a perivascular space. WMH were extracted from brain image sets using an intensity thresholding technique. $\mathrm{WMH}$ volume was corrected for total intracranial volume. Logistic and linear regression models were used to calculate odds ratios for SBI as a binary variable and $\mathrm{WMH}$ as a continuous outcome, respectively. The effects of physical activity on the outcomes were tested. Physical activity was estimated using the metabolic equivalent (MET) score. The MET score was defined as the product of the MET of the individual's activities estimated using sports and exercise compendia and the weekly duration of the activity. Statistical models were adjusted for demographic variables and vascular risk factors. 
Among 1238 participants with a mean age of $70 \pm 9$ years $16 \%$ had $\mathrm{SBI}$ and $43 \%$ were physically inactive. High intensity activity, i.e., the upper quartile of the MET score, but not low intensity activity was decreased the risk of SBI by $40 \%$, adjusted for other risk factors and demographic imbalances. Those participants that were active but had Medicaid or no insurance, however, did not benefit from exercise as much as individuals with Medicare or private insurance. No association between activity and $\mathrm{WMH}$ volume was found.

\section{Discussion}

The study supports the role of physical activity as a cerebrovascular risk factor. While several studies have suggested inactivity to be a risk factor for ischemic stroke $\{\mathrm{Hu} 2005$; Willey 2009\}, this study extends this evidence to subclinical brain infarction. Those individuals who are physically inactive are more likely to have small SBIs even in models adjusted for conventional risk factors like diabetes, cholesterol levels and hypertension. The data further show that the effect of activity depends on its intensity. Only vigorous activity (MET scores of 14 or higher, that equals to 2 hours per week of jogging at $5 \mathrm{mph}$ or swimming in laps) has a protective effect. Similarly, the Cardiovascular Health Study had shown differences between low, moderate and high intensity exercise that gradually reduced mortality in individuals 65 years or older \{Mozaffarian 2004\}.

Interestingly, the protective effects of activity were only found for subclinical infarcts and not for $\mathrm{WMH}$. This may suggest different pathophysiological mechanisms with different predisposing or triggering factors leading to $\mathrm{SBI}$ or $\mathrm{WMH}$. Hypertension, a wellcharacterized risk factors for ischemic lesions, did not predict the increase in WMH volume over time in a longitudinal MRI study \{Maillard 2009\}. In the Atherosclerosis Risk in Communities (ARIC) study, new infarcts on MRI, but not WMH, were found to be more likely in subjects with diabetes and hypertension \{Knopman 2011\}. On the other hand, small vessel stroke is associated with WMH suggesting the mechanistic link. It may be that WMH are not a sensitive endpoint for risk factor studies due to methodological difficulties with their quantification.

That insurance status - which the authors interpret as a proxy for socioeconomic status influences the protective effect of activity on SBI, is difficult to explain. One reason may be that other risk factors are present in lower socioeconomic groups for which the statistical models used in the present study were not corrected. Alternatively, lower socioeconomic groups may substitute leisure/recreational exercise (which was recorded in the study) with work-related activity that may exert similar protective effects if it is sufficiently intense. 
One limitation of the study, or strength depending on the country or region where the results are to be used, is the particular ethnic distribution with a high percentage of individuals of hispanic origin (65\%). These were less likely to be physically active as compared with others. However, the statistical models were corrected for ethnicity.

\section{Future perspective}

Physical activity and exercise programs are of fundamental importance for the elderly. Aged individuals who remain physically active have better cerebrovascular health which pertains not only to strokes but also the cumulative vascular brain damage by subclinical infarcts that causes the typical triad of age-related symptoms, gait disorders, depression, and dementia. Health care providers and family members need to encourage elderly individuals to engage in exercise. More exercise programs that are optimized for elderly participants are required.

\section{Executive summary}

- Inactivity is a risk factor for subclinical brain infarction.

- Vigorous activity but not low-intensity exercise decreases the risk for subclinical brain infarction as detected by MRI by $40 \%$.

- No effects of physical activity on cerebral white matter hyperintensities as detected by MRI were found.

- Exercise programs should be widely used in the elderly population.

\section{References}

\{bibliography\}

\section{Financial disclosure}

Dr. Luft is member of the scientific advisory board of Hocoma AG, Switzerland. The companies products are not discussed or mentioned in the manuscript. No writing assistance was utilized in the production of the manuscript. 